\title{
Modelling the evolution and nucleosynthesis of carbon-enhanced metal-poor stars
}

\author{
O. R. Pols ${ }^{1}$, R. G. Izzard ${ }^{1} \dagger$, M. Lugaro ${ }^{1}$ and S. E. de Mink ${ }^{1}$ \\ ${ }^{1}$ Sterrekundig Instituut Utrecht, P. O. Box 80000, NL-3584 TA Utrecht, The Netherlands \\ email: O.R.Pols@uu.nl, R.G.Izzard@uu.nl, M. A. Lugaro@uu.nl, S. E.deMink@uu.nl
}

\begin{abstract}
We present the results of binary population simulations of carbon-enhanced metalpoor (CEMP) stars. We show that nitrogen and fluorine are useful tracers of the origin of CEMP stars, and conclude that the observed paucity of very nitrogen-rich stars puts strong constraints on possible modifications of the initial mass function at low metallicity. The large number fraction of CEMP stars may instead require much more efficient dredge-up from low-metallicity asymptotic giant branch stars.
\end{abstract}

Keywords. Stars: AGB and post-AGB, stars: evolution, stars: binaries, stars: abundances, stars: mass function

\section{Introduction}

One of the most striking results of recent large surveys for very metal-poor stars in the Galactic halo is the large proportion of highly carbon-enriched objects among them. These carbon-enhanced metal-poor (CEMP) stars, usually defined as metal-poor stars with $[\mathrm{C} / \mathrm{Fe}]>1.0$, make up at least 10 per cent and probably as much as 20-25 per cent of very metal-poor stars with $[\mathrm{Fe} / \mathrm{H}]<-2$ (Frebel et al. 2006; Lucatello et al. 2006).

The majority (about 80 per cent, according to Aoki et al. 2007) of CEMP stars are also enriched in $\mathrm{Ba}$ and other heavy elements produced by slow neutron captures (the $s$-process) in asymptotic giant branch (AGB) stars. For these so-called CEMP-s stars a likely scenario is pollution by mass transfer from a more massive AGB companion in a binary system, which has since become a white dwarf. Supporting evidence for this scenario comes from radial velocity monitoring, which suggests that all CEMP-s stars could statistically be binaries (Lucatello et al. 2005a). The remaining fraction of CEMP stars that do not show s-process enrichments (the CEMP-no stars) are typically more metal-poor than the CEMP-s stars and so far have shown no evidence for binarity (Aoki et al. 2007). These stars exhibit a variety of abundance patterns, and may have formed instead from material ejected from rapidly rotating massive stars (Meynet et al. 2006) or from faint core-collapse supernovae (Umeda \& Nomoto 2005). Confusing such a clear distinction are a surprisingly large number of CEMP stars enhanced in both $s$-process and $r$-process (rapid neutron-capture) elements, whose origin is still quite unclear (Jonsell et al. 2006).

Within the mass transfer scenario, the large proportion of CEMP-s stars requires the existence of a sufficient number of binary systems with primary components that have undergone AGB nucleosynthesis. In recent studies (Lucatello et al. 2005b; Komiya et al. 2007) it has been argued that this requires a different initial mass function (IMF) at low metallicity, weighted towards intermediate-mass stars. If true, this in turn has important consequences for the chemical evolution of the halo and, by implication, of other galaxies.

$\dagger$ Present address: Institut d'Astronomie et d'Astrophysique, Université Libre de Bruxelles, CP226, Boulevard du triomphe, B-1050 Bruxelles, Belgium 
However, the model calculations on which these estimates are based still contain many uncertainties regarding the evolution and nucleosynthesis of low-metallicity AGB stars, the efficiency of mass transfer, and the evolution of the surface abundances of the CEMP stars themselves.

In this contribution we explore the effect of some of these uncertainties on the number fraction of CEMP stars by means of a binary population synthesis study. Apart from carbon, we concentrate on nitrogen and fluorine enrichments as possible tracers of the origin of CEMP stars, the latter motivated by the recent discovery of fluorine in a CEMP star (see Sect. 2). In Sect. 3 we present the first results of our binary population synthesis simulations, and in Sect. 4 we give our conclusions.

\section{Nitrogen and fluorine in CEMP stars}

Apart from carbon, substantial enhancements of nitrogen with respect to iron are common among CEMP stars, typically with $[\mathrm{C} / \mathrm{N}]>0$. Detailed AGB nucleosynthesis models of low initial mass $\left(<2.5 M_{\odot}\right)$ produce carbon (by the $3 \alpha$ reaction during thermal pulses and subsequent convective dredge-up), but do not produce nitrogen because it is burned during the same helium shell flashes. On the other hand, AGB models of higher mass convert the dredged-up carbon effectively into nitrogen by $\mathrm{CN}$-cycling at the bottom of the convective envelope (hot bottom burning, HBB). The surface abundances of these more massive AGB stars approach the $\mathrm{CN}$-equilibrium ratio of $[\mathrm{C} / \mathrm{N}] \approx-2$. Detailed evolution models of AGB stars (Karakas \& Lattanzio 2007) indicate that HBB sets in at significantly lower mass at low metallicity (between 2.5 and $3 M_{\odot}$ at $[\mathrm{Fe} / \mathrm{H}]=$ -2.3 ) than at solar metallicity (around $5 M_{\odot}$ ). One may thus expect a population of so-called nitrogen-enhanced metal-poor (NEMP) stars, with $[\mathrm{C} / \mathrm{N}]<-0.5$. Although a few examples of such stars are known, mostly at $[\mathrm{Fe} / \mathrm{H}]<-2.9$, they appear to be very rare (Johnson et al. 2007). As we show in Sect. 3 the number of NEMP stars sets an additional constraint on possible changes to to IMF at low metallicity.

Recently, Schuler et al. (2007) derived a super-solar fluorine abundance of $[\mathrm{F} / \mathrm{Fe}]=$ +2.9 in the halo CEMP star HE $1305+0132$. This is the most iron-deficient star, $[\mathrm{Fe} / \mathrm{H}]=$ -2.5 , for which the fluorine abundance has been measured. Enhancements of carbon and nitrogen are also measured $([\mathrm{C} / \mathrm{Fe}]=+2.7 ;[\mathrm{N} / \mathrm{Fe}]=+1.6)$, and $\mathrm{Ba}$ and $\mathrm{Sr}$ lines are seen in its spectrum (Goswami 2005), placing HE 1305+0132 in the group of CEMP-s stars.

Fluorine can be made in AGB stars as a by-product of the ${ }^{14} \mathrm{~N}+{ }^{4}$ He reaction under neutron-rich conditions during thermal pulses (Lugaro et al. 2004). Fluorine enhancements of up to 30 times solar have been measured among Galactic AGB stars (Jorissen et al. 1992), demonstrating that these stars can indeed produce fluorine efficiently. Detailed AGB nucleosynthesis models (Karakas \& Lattanzio 2007) show that fluorine is produced and dredged to the surface alongside carbon in low-metallicity AGB stars with masses $<3 M_{\odot}$. At larger masses fluorine is destroyed by proton captures as a result of HBB.

In Fig. 1 we show the enhancements of $\mathrm{F}$ and $\mathrm{C}+\mathrm{N}$ relative to hydrogen as observed in HE $1305+0132$ and compare these to the abundance ratios in the material lost by AGB stars at $[\mathrm{Fe} / \mathrm{H}]=-2.3$ according to the Karakas \& Lattanzio (2007) models. The figure shows that AGB stars with masses between 1.7 and $2.3 M_{\odot}$ produce fluorine and carbon in the right amounts to account for the observed abundances, after accretion of the material by a low-mass companion and subsequent dilution in its envelope by a factor 6 to 9 (Lugaro et al. 2008). Assuming a mass of $0.8 M_{\odot}$ and a convective envelope mass fraction of $60 \%$, this implies that the star should have accreted $0.05-0.12 M_{\odot}$ from its companion, corresponding to $3-11 \%$ of the mass lost by the AGB star. These constraints 


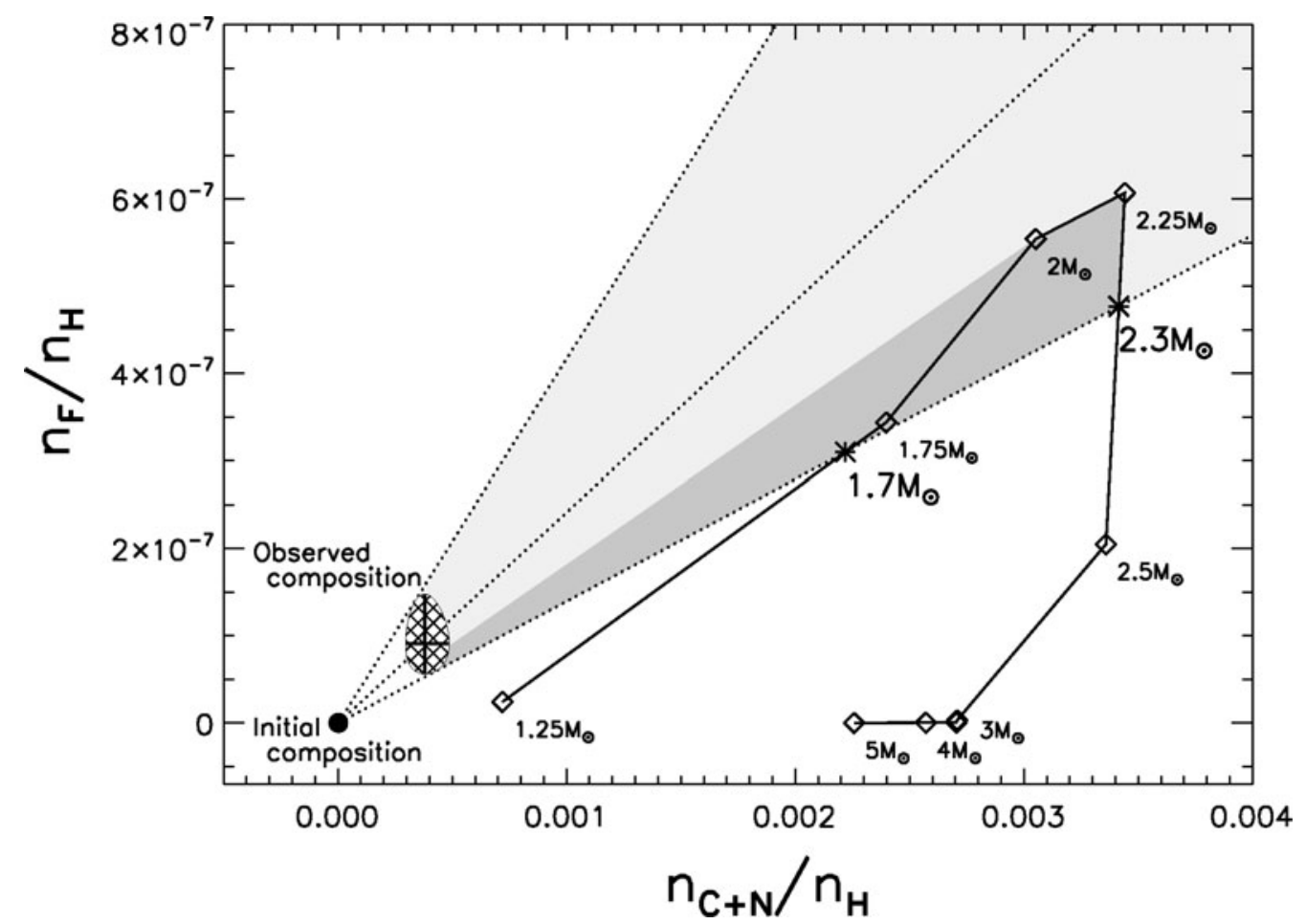

Figure 1. Abundances by number of $\mathrm{F}$ and $\mathrm{C}+\mathrm{N}$ with respect to $\mathrm{H}$ as observed in $\mathrm{HE} 1305+0132$ from Schuler et al. (2007; hatched ellipsoid showing $1 \sigma$ errors) compared to those computed in the material lost in $\mathrm{AGB}$ star winds at $[\mathrm{Fe} / \mathrm{H}]=-2.3$, with labels indicating the initial masses. The light gray area is the region of the plot where the $\mathrm{F}$ and $\mathrm{C}+\mathrm{N}$ abundances from the AGB companion should lie in order to match the observed abundances, after dilution. The darker gray area represents the region covered by the models with masses in the range $1.7-2.3 M_{\odot}$ indicated by the asterisks.

fit well within the binary mass transfer scenario. Furthermore this result indicates the potential of using fluorine as a tracer for the origin of CEMP stars.

\section{Binary population nucleosynthesis of CEMP stars}

We have simulated populations of metal-poor halo stars in binary systems using the rapid synthetic binary nucleosynthesis code of Izzard et al. (2004) and Izzard et al. (2006). The code uses fits to detailed single-star evolution and nucleosynthesis models, in particular the AGB models of Karakas \& Lattanzio (2007), and follows the surface abundances as a star evolves through dredge-up episodes. A prescription for hot bottom burning in massive AGB stars is included, calibrated against the same detailed AGB models. Binary evolution is followed according to the prescriptions of Hurley et al. (2002). We model mass transfer by stellar wind accretion, according to the Bondi \& Hoyle (1944) prescription, and by Roche-lobe overflow (RLOF). In binaries with AGB primaries, RLOF is usually unstable and leads to the ejection of a common envelope without any further accretion onto the companion. In our default model we assume efficient thermohaline mixing, in accordance with the findings of Stancliffe et al. (2007).

In all models we use solar-scaled initial abundances according to Anders \& Grevesse (1989) with $Z=10^{-4}$, corresponding to $[\mathrm{Fe} / \mathrm{H}]=-2.3$, which is the lowest metallicity for which we have detailed AGB models. We select stars with ages between 10 and $13.7 \mathrm{Gyr}$ 
Table 1. Number fractions of CEMP and NEMP stars among halo stars at $[\mathrm{Fe} / \mathrm{H}]=-2.3$ and $\log g<4.0$ resulting from binary population synthesis, for different sets of physical ingredients in the evolution models. The last two columns give the fraction of CEMP-s stars and of fluorine-rich FEMP stars, relative to the number of CEMP stars.

\begin{tabular}{llrcccc}
\hline model & $f($ CEMP $)$ & $f($ NEMP $)$ & NEMP & CEMP - CEMP & FEMP & CEMP \\
\hline 1A & default physics & $2.30 \%$ & $0.35 \%$ & 0.15 & 0.29 & 0.85 \\
2A & no thermohaline mixing & $4.20 \%$ & $0.52 \%$ & 0.12 & $\ldots$ & $\ldots$ \\
3A & calibrated 3DUP & $9.43 \%$ & $0.34 \%$ & 0.036 & 0.99 & 0.82 \\
4A & no therm. mix. + calibr. 3DUP & $14.96 \%$ & $0.47 \%$ & 0.031 & 0.75 & 0.87 \\
\hline
\end{tabular}

(roughly corresponding to the age of the halo) and $\log g<4.0$ (thus including turnoff stars and (sub)giants but excluding unevolved main-sequence stars). Among this sample we designate as CEMP stars those with $[\mathrm{C} / \mathrm{Fe}]>1.0$ and as NEMP stars those with $[\mathrm{N} / \mathrm{Fe}]>0.5$ and $[\mathrm{C} / \mathrm{N}]<-0.5$, following the definition of Johnson et al. (2007). Note that these definitions partly overlap.

We can compare our model results with the statistics of the SAGA database of metalpoor stars (Suda et al. 2008). We selected 375 stars from the database in a metallicity range $[\mathrm{Fe} / \mathrm{H}]=-2.3 \pm 0.5$ and $\log g<4.0$. Of these, 296 have a $\mathrm{C}$ abundance measurement and 69 classify as CEMP stars, yielding a CEMP fraction of 18-23\%. Only one star classifies as a NEMP star, giving a very small nominal NEMP fraction of $<0.3 \%$. If we consider an extended metallicity range $-4<[\mathrm{Fe} / \mathrm{H}]<-2$ in order to improve the number statistics, we find 6 NEMP stars and a NEMP fraction of about $1.5 \%$. We conclude that CEMP stars outnumber NEMP stars by at least a factor 10 .

In our default model (1A) we assume that the initial primary masses $M_{1}$ are distributed according to the solar neighbourhood IMF as derived by Kroupa et al. (1993), the initial periods come from a flat distribution in $\log P$ and the initial mass ratios from a flat distribution in $q=M_{2} / M_{1}$. In Table 1 we present the resulting number fractions of CEMP and NEMP stars (first row). This model clearly fails to account for the large observed CEMP fraction, although the small NEMP fraction is consistent with the observations. The last two columns give the number ratio of CEMP-s stars (defined as those CEMP stars with $[\mathrm{Ba} / \mathrm{Fe}]>0.5)$ to CEMP stars and the ratio of FEMP stars (defined as stars with $[\mathrm{F} / \mathrm{Fe}]>1.0)$ to CEMP stars. Although all our CEMP stars are also enriched in $\mathrm{Ba}$, in this model the majority have $[\mathrm{Ba} / \mathrm{Fe}]<0.5$.

In the next rows of Table 1 we vary some of the uncertain physical ingredients in our models, while keeping the input distributions the same. In model $2 \mathrm{~A}$ we switch off thermohaline mixing, which has the effect of increasing the surface abundances of $\mathrm{C}$, $\mathrm{N}$ and $\mathrm{Ba}$ in turnoff stars with shallow convection zones relative to our default model. The numbers of CEMP stars and NEMP stars correspondingly increase, although the CEMP fraction remains too low to be compatible with observations. In model $3 \mathrm{~A}$ we vary some of the parameters relating to third dredge-up (3DUP), in particular we assume a smaller value (by up to $0.1 M_{\odot}$ ) of the minimum core mass at which 3 DUP occurs, and after dredge-up has started we assume efficient 3DUP (with $\lambda \geqslant 0.8$ ) regardless of how small the envelope mass is. In practice this means that almost all AGB stars with initial masses $>0.8 M_{\odot}$ undergo efficient dredge-up. These modifications are motivated by similar changes needed to reproduce the carbon-star luminosity function of the Magellanic Clouds (Izzard \& Tout 2004) and the abundances of Galactic post-AGB stars (Bonačić Marinović et al. 2007). This leads to an increase of the CEMP fraction to almost $10 \%$, much closer to but still short of the observed value. Model $4 \mathrm{~A}$ is a combination of $2 \mathrm{~A}$ and $3 \mathrm{~A}$ and results in a CEMP fraction of $15 \%$. We note that in these latter two models 
Table 2. Number fractions of CEMP and NEMP stars among halo stars at $[\mathrm{Fe} / \mathrm{H}]=-2.3$ and $\log g<4.0$ as in Table 1 , for the default physical ingredients while varying the input distributions.

\begin{tabular}{llrrr}
\hline model & & $f($ CEMP $)$ & $f($ NEMP $)$ & NEMP \\
\hline CEMP & default $N\left(M_{1}, q, P\right)$ & $2.30 \%$ & $0.35 \%$ & 0.15 \\
1B & $N(q, P)$ from Duquennoy \& Mayor $(1991)$ & $3.50 \%$ & $0.71 \%$ & 0.20 \\
1C & $N\left(M_{1}\right)$ from Miller \& Scalo $(1979)$ & $3.15 \%$ & $0.62 \%$ & 0.20 \\
1D & $N\left(M_{1}\right)$ from Lucatello et al. $(2005 \mathrm{~b})$ & $4.81 \%$ & $1.35 \%$ & 0.28 \\
1E & $N\left(M_{1}\right)$ from Komiya et al. $(2007)$ & $13.47 \%$ & $26.61 \%$ & 1.98 \\
\hline
\end{tabular}

nearly all CEMP stars are CEMP-s stars, in accordance with observations. We also note that in all models the majority $(>80 \%)$ of CEMP stars are expected to be fluorine-rich, with $[\mathrm{F} / \mathrm{Fe}]>1.0$.

In Table 2 we present CEMP and NEMP fractions of the default physical model while varying the initial distributions of binary parameters. In model $1 \mathrm{~B}$ we assume mass ratios and periods drawn from the distributions derived by Duquennoy \& Mayor (1991) for the local population of $\mathrm{G}$ dwarfs (i.e. a log-normal period distribution with a broad peak at 170 years and a $q$ distribution with a broad peak at $M_{2} / M_{1}=0.23$ ). This leads to an increase by a factor of 1.5-2 in the number of CEMP and NEMP stars as the peak in the period distribution coincides with the period range in which mass transfer is effective.

Models 1C, 1D and 1E explore the effect of varying the initial mass function, by assuming the default $q$ and $P$ distributions in combination with a log-normal form of the IMF. The Miller \& Scalo (1979) IMF also represents the solar neighbourhood but gives somewhat higher CEMP and NEMP fractions than the Kroupa et al. (1993) IMF. Model 1D assumes the IMF suggested by Lucatello et al. (2005b) as required to reproduce the large CEMP fraction (it has a median mass of $0.79 M_{\odot}$, compared to $0.10 M_{\odot}$ for the Miller \& Scalo IMF). It results in a larger CEMP fraction but still falls short of the observed value. The discrepancy between our and Lucatello's results arises mainly because in our (default) models the initial primary mass and period range contributing to CEMP stars are smaller than they assumed. Model 1D also shows an increased NEMP fraction, the result of a larger weight of intermediate-mass stars (with $M>2.7 M_{\odot}$ undergoing $\mathrm{HBB}$ ) in this IMF. This effect is much more extreme when we assume the IMF suggested by Komiya et al. (2007) which has a median mass of $10 M_{\odot}$. Although it gives rise to a substantial CEMP fraction, the CEMP stars are outnumbered by NEMP stars by a factor of two. This is not compatible with the observed limits on the number fraction of NEMP stars.

\section{Conclusions}

The detection of a large fluorine overabundance in the CEMP star HE 1305+0132 is well explained within the AGB binary mass transfer scenario (Lugaro et al. 2008). On the other hand, models of rapidly rotating massive stars do not produce fluorine (Meynet et al. 2006). Therefore fluorine appears to be a useful discriminant between different scenarios proposed for the origin of CEMP stars. Our population synthesis results indicate that (rather independent of model assumptions) at least $80 \%$ of CEMP stars formed by AGB mass transfer should be enriched in fluorine $([\mathrm{F} / \mathrm{Fe}]>1.0)$, i.e. most CEMP-s stars should also be FEMP stars.

Our binary population synthesis models show that the paucity of NEMP stars among metal-poor halo stars is incompatible with a strongly modified IMF at low metallicity, 
heavily weighted towards intermediate-mass stars, as has been suggested by Komiya et al. (2007) in order to explain the high proportion of CEMP stars. Another possible explanation for the ubiquity of CEMP-s stars is that low-metallicity AGB stars undergo much more efficient dredge-up than shown by the detailed evolution models available to date.

\section{Acknowledgements}

We would like to thank Takuma Suda for making the SAGA database of metal-poor stars available to us in electronic form ahead of publication.

\section{References}

Anders, E. \& Grevesse, N. 1989, Geochim. Cosmochim. Acta 53, 197

Aoki, W., Beers, T. C., Christlieb, N., Norris, J. E., Ryan, S. G., \& Tsangarides, S. 2007, ApJ 655,492

Bonačić Marinović, A., Izzard, R. G., Lugaro, M., \& Pols, O. R. 2007, A\&A 469, 1013

Bondi, H., \& Hoyle, F. 1944, MNRAS 104, 273

Duquennoy, A., \& Mayor, M. 1991, A\&A 248, 485

Frebel, A., Christlieb, N., Norris, J. E., et al. 2006, ApJ 652, 1585

Goswami, A. 2005, MNRAS 359, 531

Hurley, J. R., Tout, C. A., \& Pols, O. R. 2002, MNRAS 329, 897

Izzard, R. G., \& Tout, C. A. 2004, MNRAS 350, L1

Izzard, R. G., Tout, C. A., Karakas, A. I., \& Pols, O. R. 2004, MNRAS 350, 407

Izzard, R. G., Dray, L. M., Karakas, A. I., \& Lugaro, M., \& Tout, C. A. 2006, A\&্A 460, 565

Johnson, J. A., Herwig, F., Beers, T. C., \& Christlieb, N. 2006, ApJ 658, 1203

Jonsell, K., Barklem, P. S., Gustafsson, B., Christlieb, N., Hill, V., Beers, T. C., \& Holmberg, J. 2006, ApJ 451, 651

Jorissen, A., Smith, V. V., \& Lambert, D.L. 1992, A\&SA 261, 164

Karakas, A. \& Lattanzio, J. C. 2007, PASA 24, 103

Komiya, Y., Suda, T., Minaguchi, H., Shigeyama, T., Aoki, W., \& Fujimoto, M. Y. 2007, ApJ 658, 367

Kroupa, P., Tout, C. A., \& Gilmore, G. 1993, MNRAS 262, 545

Lucatello, S., Tsangarides, S., Beers, T. C., Carretta, E., Gratton, R. G., \& Ryan, S. G. 2005, ApJ 625, 825

Lucatello, S., Gratton, R. G., Beers, T. C., \& Carretta, E. 2005, ApJ 625, 833

Lucatello, S., Beers, T. C., Christlieb, N., Barklem, P. S., Rossi, S., Marsteller, B., Sivarani, T., \& Lee, Y. S. 2006, ApJ 652, L37

Lugaro, M., Ugalde, C., Karakas, A. I., Görres, J., Wiescher, M., Lattanzio, J. C., \& Cannon, R. C. 2004, ApJ, 615, 934

Lugaro, M., de Mink, S. E., Izzard, R. G., et al. 2008, A\&3A 484, L27

Meynet, G., Ekström, S., \& Maeder, A. 2006, A\&A 447, 623

Miller, G. E., \& Scalo, J. M. 1979, ApJS 41, 513

Schuler, S. C., Cunha, K., Smith, V. V., Sivarani, T., Beers, T. C., \& Lee, Y. S. 2007, ApJ 667, L81

Stancliffe, R. J., Glebbeek, E., Izzard, R. G., \& Pols, O. R. 2007, A $\& A$ 464, L57

Suda, T., Katsuta, Y., Yamada, S., et al. 2008, PASJ 60, in press, arXiv:0806.3697

Umeda, H. \& Nomoto, K. 2005, ApJ 619, 427

\section{Discussion}

C. Charbonnel: You spoke about observations of s-process elements in CEMP in general. Are there some precise data, regarding the low- and high-mass s-elements, that may help discriminate the progenitor (e.g., AGB vs massive stars)? 
O. Pols: There are certainly many detailed abundance determinations of heavy elements in CEMP stars, and these have been tested against s-process models by several groups. In general, these observations agree with AGB models, although different assumptions must be made for different stars regarding the free parameters in these models (such as the ${ }^{1} 3 C$ pocket size), and there are still many open questions in this respect. Some CEMP stars show both an s-process and r-process signature, for these one may need pre-enrichment by massive stars and AGB mass transfer...

N. LANGER: Could the fluorine in CEMP stars also come from neutrino-induced nucleosynthesis from supernovae?

O. Pols: Indeed, supernovae can also be sources of fluorine although this has not been confirmed observationally to my knowledge. However, SNe typically produce more Oxygen than Carbon, contrary to what is seen in CEMP stars. One would need to get rid of the Oxygen, or suppose a different source for the carbon. AGB stars naturally explain both $\mathrm{C}$ and $\mathrm{F}$ enhancements simultaneously. 